MITSUBISHI ELECTRIC RESEARCH LABORATORIES

http://www.merl.com

\title{
Inter-Band Carrier Aggregation Digital Transmitter Architecture with Concurrent Multi-Band Delta-Sigma Modulation Using Out-of-Band Noise Cancellation
}

\author{
Chung, S.W.; Ma, R. \\ TR2015-043 May 2015
}

\begin{abstract}
Concurrent multi-band delta-sigma modulation (CMB-DSM) with out-of-band noise cancellation is presented for the first time. A scaled replica modulator creates anti-phased quantization noise for multi-band noise cancellation, consequently reducing adjacent channel leakage and significantly relaxing the design of a multi-band RF filter to suppress undesirable out-ofband emission. A prototype digital transmitter is designed with 6th-order CMB-DSM using a loop filter with stability compensation. Experimental results show that the proposed noise cancellation technique achieves more than 15- dB suppression on the out-of-band quantization noise with LTE inter-band carrier aggregation (CA) on $856 \mathrm{MHz}$ and $1450 \mathrm{MHz}$ bands with 40-MHz aggregated channel bandwidth.
\end{abstract}

2015 International Microwave Symposium (IMS)

This work may not be copied or reproduced in whole or in part for any commercial purpose. Permission to copy in whole or in part without payment of fee is granted for nonprofit educational and research purposes provided that all such whole or partial copies include the following: a notice that such copying is by permission of Mitsubishi Electric Research Laboratories, Inc.; an acknowledgment of the authors and individual contributions to the work; and all applicable portions of the copyright notice. Copying, reproduction, or republishing for any other purpose shall require a license with payment of fee to Mitsubishi Electric Research Laboratories, Inc. All rights reserved. 



\title{
Inter-Band Carrier Aggregation Digital Transmitter Architecture with Concurrent Multi-Band Delta-Sigma Modulation Using Out-of-Band Noise Cancellation
}

\author{
SungWon Chung ${ }^{1,2}$, Rui Ma ${ }^{1}$, Shintaro Shinjo ${ }^{3}$, Koon H. Teo ${ }^{1}$ \\ ${ }^{1}$ Mitubishi Electric Research Laboratories, Cambridge, MA, USA, rma@merl.com \\ ${ }^{2}$ Massachusetts Institute of Technology, Cambridge, MA, USA \\ ${ }^{3}$ Mitsubishi Electric Corporation, Information Technology R\&D Center, Kamakura, Japan
}

\begin{abstract}
Concurrent multi-band delta-sigma modulation (CMB-DSM) with out-of-band noise cancellation is presented for the first time. A scaled replica modulator creates anti-phased quantization noise for multi-band noise cancellation, consequently reducing adjacent channel leakage and significantly relaxing the design of a multi-band RF filter to suppress undesirable out-of-band emission. A prototype digital transmitter is designed with 6th-order CMB-DSM using a loop filter with stability compensation. Experimental results show that the proposed noise cancellation technique achieves more than 15dB suppression on the out-of-band quantization noise with LTE inter-band carrier aggregation $(\mathrm{CA})$ on $856 \mathrm{MHz}$ and $1450 \mathrm{MHz}$ bands with 40-MHz aggregated channel bandwidth.

Index Terms-Digital transmitter, concurrent multi-band delta-sigma modulation, inter-band carrier aggregation.
\end{abstract}

\section{INTRODUCTION}

For the efficient utilization of available spectrum, concurrent multi-band transmission techniques have been developed such as intra-band and inter-band carrier aggregation (CA) for LTE-Advanced. In practice, inter-band CA demands more sophisticated radio implementation compared to the intra-band case. An important design challenge with the inter-band CA transmitters lies in the RF power amplifier (PA), whose radio performance normally needs to be compromised to support widely separated bands when a single PA is used. Compared to the analog PAs using concurrent multi-band matching network [1], all-digital transmitters for inter-band CA [2-4] (Fig. 1) allow more compact and flexible design by using switch-mode Class-S PAs, becoming an enabler for SDR (software defined radio). Moreover, there is recently a growing interest towards digital transmitters for inter-band CA because of the progress made on improving the power efficiency of digital transmitters with multi-level operation [5], [6].

Among the inter-band CA digital transmitters, concurrent multi-band delta-sigma modulation (CMB-DSM) [2], [4] provides unique advantages that less spurious tones are generated compared to pulse-width modulation (PWM) technique [6]. Nevertheless, CMB-DSM adds a large amount of out-of-band quantization noise to the transmitter output. Thus, an additional high-Q multi-band RF filter is necessary to

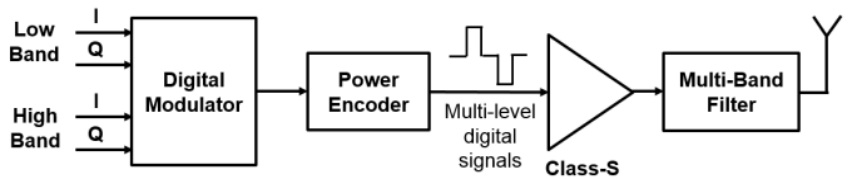

Fig. 1. General all-digital transmitter architecture for inter-band carrier aggregation (CA).

suppress those undesirable out-of-band noises [7]. Unfortunately, high-Q multi-band RF filters are notorious for overly sophisticated design, large insertion loss as well as bulky volume.

In this paper, we propose an inter-band CA digital transmitter architecture based on multi-level CMB-DSM with out-of-band noise cancellation. Out-of-band quantization noise from CMB-DSM is suppressed by adding cancellation signal at the transmitter output using an asymmetric power combiner. Consequently, the design requirement on a multi-band RF filter to suppress the out-of-band emission is significantly relaxed. Out-of-band noise cancellation also reduces the adjacent channel leakage that is hard to be filtered.

\section{INTER-BAND CA DIGITAL TRANSMITTER ARCHITECTURE}

\section{A. Concurrent Multi-Band Delta-Sigma Modulation}

Fig. 2 shows a prototype inter-band CA digital transmitter architecture based on the CMB-DSM with out-of-band noise cancellation. This dual-band implementation can be extended for general multi-band CA. Baseband quadrature signal for frequency band $k$ is denoted as a pair of $I_{k}$ and $Q_{k}$. Each pair of the quadrature baseband signal is modulated to each frequency band $\omega_{k}$ by a quadrature digital up-conversion mixer. The CMB-DSM output generates a multi-level digital signal driving a multi-level digital Class-S PA. The out-ofband quantization noise and high-order image spectra from DSM are filtered by the multi-band RF filter.

The out-of-band noise cancellation in Fig. 2 is performed by adding a noise cancellation signal, which is typically 6-dB below the main PA output power, to the transmitter output by using a power combiner with an asymmetric combining ratio. 


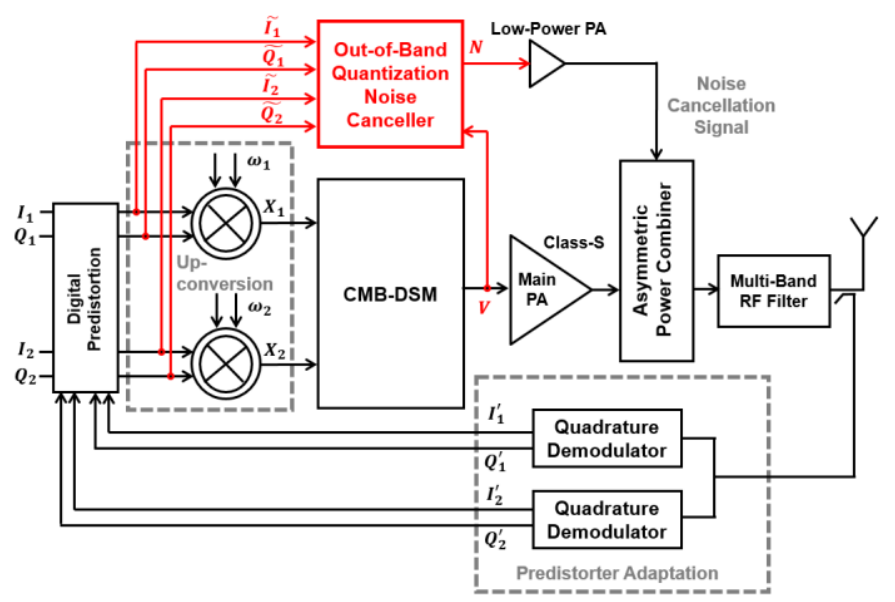

Fig. 2. Prototype inter-band carrier aggregation (CA) digital transmitter architecture based on concurrent multi-band delta-sigma modulation (CMB-DSM) with out-of-band noise cancellation.

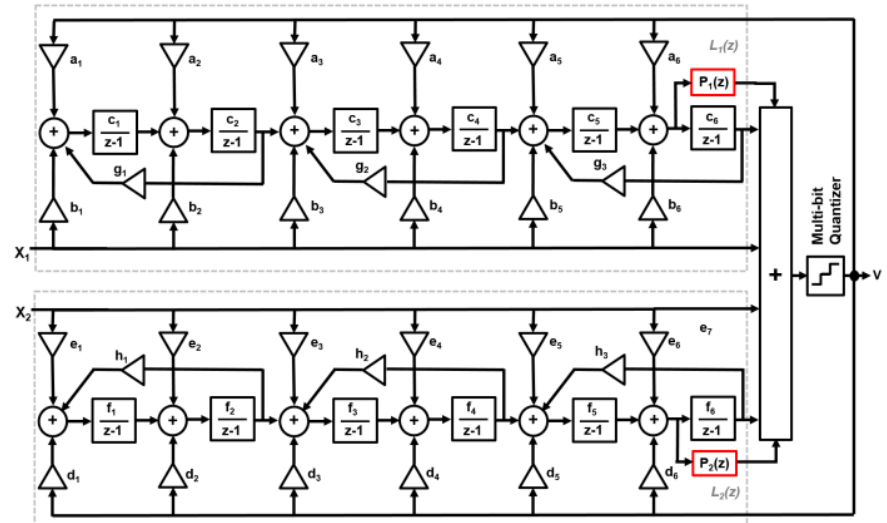

Fig. 3. 6th-order CMB-DSM design of the prototype inter-band CA digital transmitter, with loop-filter compensation for improved stability.

Reduced out-of-band noise allows a relaxed multi-band RF filter design. The filter output is coupled by a directional coupler and used by adaptive digital predistortion subsystem to characterize the transmitter nonlinearity whose impact is different for each frequency band; therefore two quadrature demodulators separately capture each frequency band.

Fig. 3 shows the $6^{\text {th }}$ order CMB-DSM design in the prototype inter-band CA digital transmitter, which includes one 7-level quantizer and two digital RF inputs for concurrent dual-band transmission. When the frequency gap with interband CA is small, high peak may occur in the noise transfer function, which degrades the modulator stability with conventional CMB-DSM implementations such as shown in [4], [7]. In order to improve the modulator stability, we added the loop-filter compensation $P_{k}(z)$ to provide a complexconjugate pole pair that reduces the peaking of the noise transfer function. Though digital predistortion can be integrated within CMB-DSM using a feedback predistortion

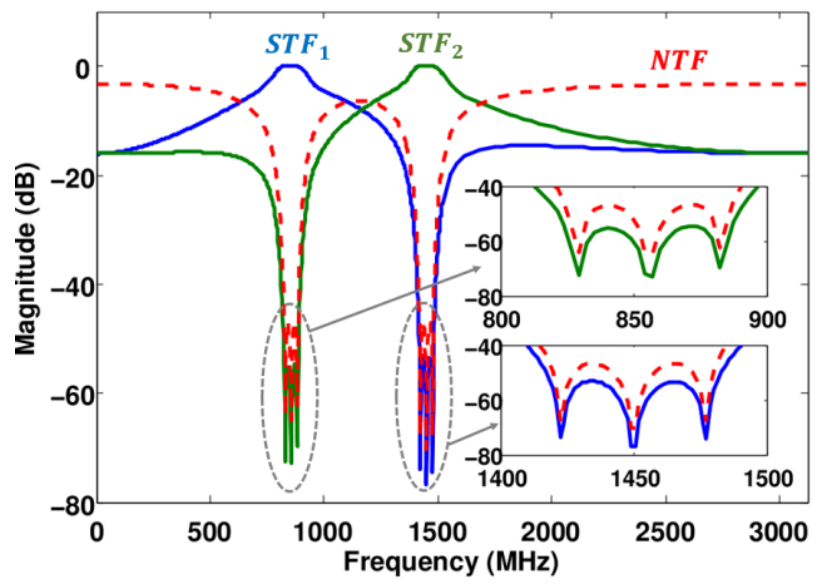

Fig. 4. Simulated noise and signal transfer function of the $6^{\text {th }}$-order CMB-DSM with $6.25-\mathrm{GHz}$ sampling rate for dual-band transmission at $856 \mathrm{MHz}$ and $1450 \mathrm{MHz}$ frequency bands.

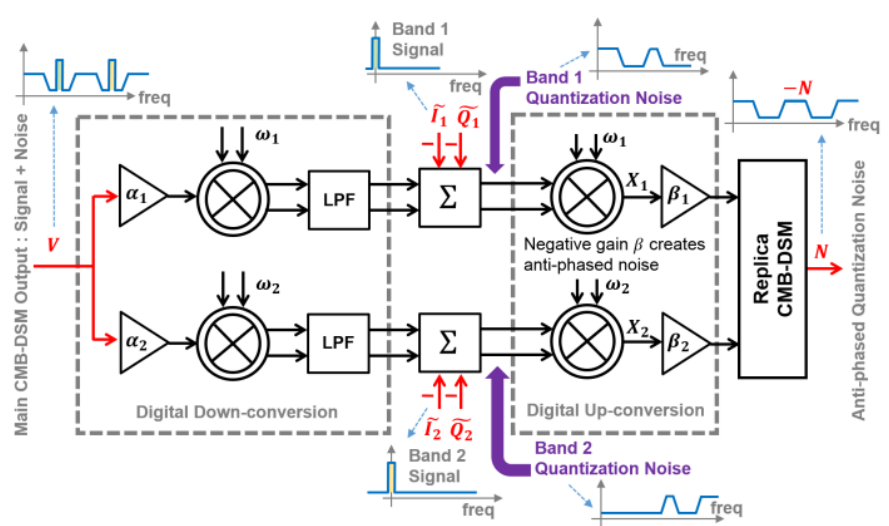

Fig. 5. Out-of-band noise canceller in the prototype CMB-DSM digital transmitter (Fig. 2), which creates the anti-phased quantization noise by subtracting the reference baseband signal in each band from the CMB-DSM output.

technique such as [8], to simplify out-of-band noise cancellation, digital predistortion is not covered in CMBDSM.

Fig. 4 shows the simulated signal and noise transfer function of the $6^{\text {th }}$ order CMB-DSM design, which has two pass-bands at $856 \mathrm{MHz}$ and $1450 \mathrm{MHz}$ with $6.25-\mathrm{GHz}$ modulator sampling rate. The two loop filters are designed to provide better than 40-dB dynamic range. The poles of each loop filter are placed around the center frequency of each frequency band. Thus the signal transfer function between each input and the output for a frequency band $k$, which is derived in [4] as $\operatorname{STF}_{k}(z)=\left(1+L_{k}(z)\right) /\left(1+\sum_{i} L_{i}(z)\right)$, becomes close to unity in each frequency band as the magnitude of $L_{k}(z)$ gets large. The noise transfer function is obtained as $\operatorname{NTF}(z)=1 /\left(1+\sum_{i} L_{i}^{\prime}(z)+\sum_{i} P_{i}(z)\right)$ where $L_{i}^{\prime}(z)$ is for the loop-filters before compensation, explaining 


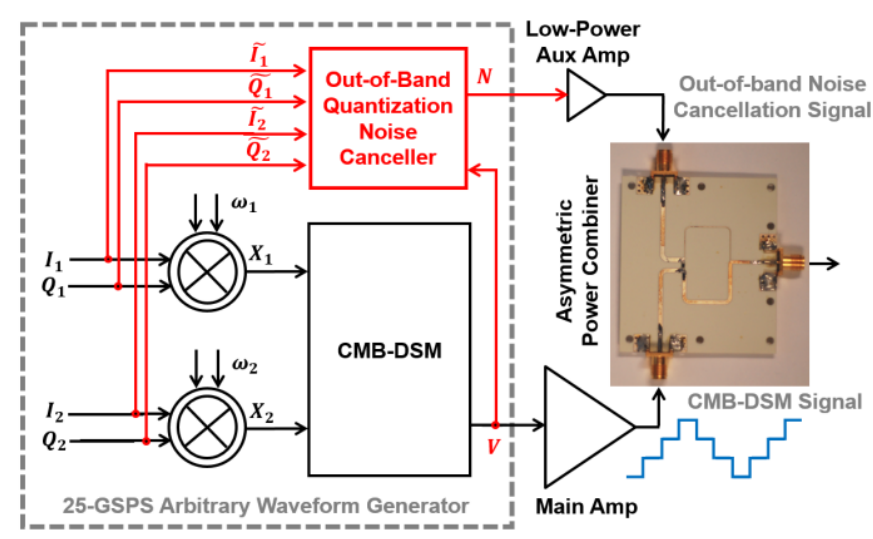

Fig. 6. Experimental CMB-DSM transmitter using out-of-noise cancellation for LTE inter-band CA with $40-\mathrm{MHz}$ bandwidth.

that the noise transfer function (Fig. 4) does not show a high peaking and shows three nulls in each pass band.

\section{B. Out-of-Band Quantization Noise Cancellation}

Fig. 5 shows the proposed out-of-band quantization noise canceller for CMB-DSM, which produces anti-phased noise. Because CMB-DSM has multiple input and a single output, the out-of-band noise cannot be determined by direct one-toone comparison such as demonstrated in [9]. Instead, the antiphased quantization noise is created by comparing the CMBDSM output with the two reference baseband signals for dualband transmission.

To produce the anti-phased noise, the quadrature digital demodulation on the CMB-DSM output $V$ is performed (Fig. 5 ), producing the sum of signal and noise for each band. The digital low-pass filters after the quadrature demodulators are for band selection. Then the out-of-band noise in each band is obtained by subtracting each reference baseband signal from each of the demodulated signals. The anti-phased out-of-band DSM quantization noise $N$ is generated by a replica CMBDSM (Fig. 5) whose two inputs are scaled by a negative gain of $\beta$. The nominal value of $\beta$ makes the maximum power of the noise cancellation signal power to be the same as the lowest non-zero output power of the main PA. If the number of output levels with the main PA is given by $\mathrm{L}$ and the output levels are uniformly spaced, the nominal value of $\beta$ reduces the out-of-band noise by up to L times.

The power of the anti-phased noise cancellation signal, which depends on the value of $\beta$, is lower than the main signal. Therefore an asymmetric power combiner (Fig. 2) is used to combine the main PA signal with the out-of-band noise cancellation signal with high combining efficiency. Although symmetric power combiners with equal input power ratio can be also used for the out-of-band noise cancellation, the combining efficiency is degraded when the two input ports receive unequal power.

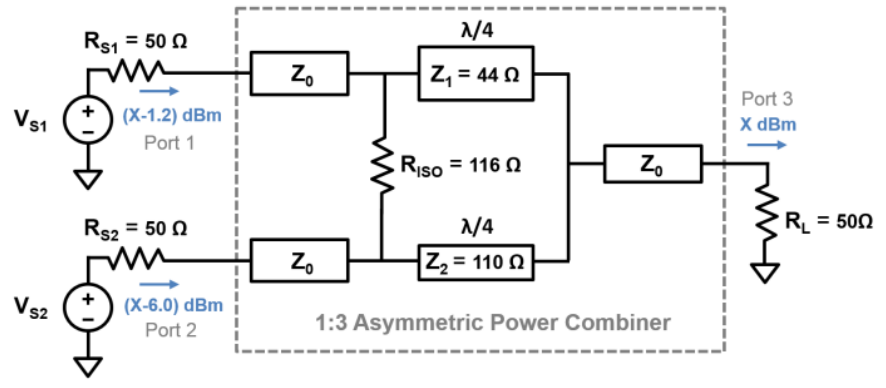

(a)

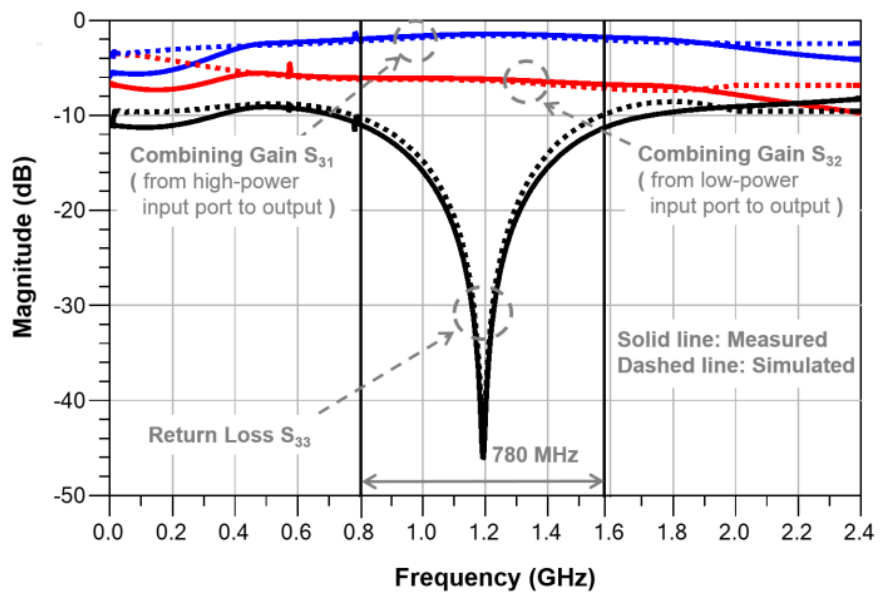

(b)

Fig. 7. Asymmetric power combiner for the experimental prototype: (a) design with 1:3 combining ratio, (b) measured characteristics.

\section{MEASURED RESULTS}

Fig. 6 shows the proof-of-concept prototype inter-band CA digital transmitter, which is implemented with a $25-\mathrm{GS} / \mathrm{s}$ arbitrary waveform generator (AWG), Hittite HMC870LC5 wideband amplifiers imitating 7-level digital Class-S PAs, and an asymmetric RF power combiner. Both CMB-DSM output signal and out-of-band noise cancellation signal for LTE interband CA with $40-\mathrm{MHz}$ aggregate bandwidth are precalculated by software and downloaded into the AWG.

Fig. 7 shows the asymmetric power combiner with an unequal input power ratio of 1:3 for the prototype transmitter. The asymmetric power combiner design is based on [10] and simplified for small size by compromising bandwidth. The measured characteristics in Fig. 7(a) show the insertion loss below $1 \mathrm{~dB}$ and the bandwidth of $0.8 \mathrm{GHz}$.

Fig. 8 shows the measured dual-band spectrum of the prototype transmitter before the multi-band RF filter input, exhibiting more than 15-dB out-of-band noise suppression. To relax the high-order design requirement on a multi-band RF filter, the most desirable out-of-band noise suppression can be obtained by adjusting the gain factors of the noise canceller (Fig. 5). Without the noise cancellation, the in-band channel power decreases since the insertion loss of the asymmetric power combiner increases when the noise cancellation input is 


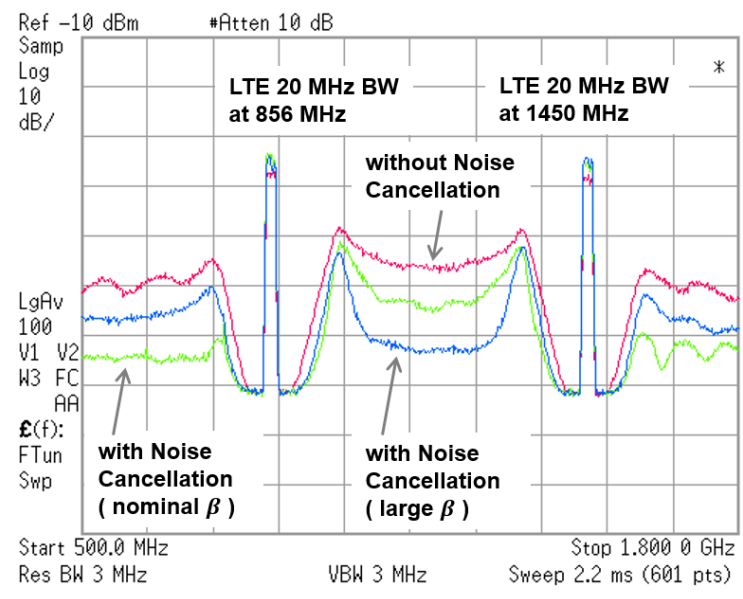

Fig. 8. Measured output spectrum of the prototype CMB-DSM digital transmitter with LTE inter-band CA on $40-\mathrm{MHz}$ aggregate bandwidth at $856 \mathrm{MHz}$ and $1450 \mathrm{MHz}$ channels.

not presented to the combiner. If the power of the noise cancellation signal is too low, the cancellation is not sufficient. If the cancellation signal power is too high, the quantization noise of the cancellation signal itself becomes comparable to the quantization noise of the main signal, which also limits the noise cancellation performance.

Fig. 9 shows the measured wideband spectrum with the LTE inter-band CA with 40-MHz aggregate bandwidth at 856 $\mathrm{MHz}$ and $1450 \mathrm{MHz}$. The $6.25-\mathrm{GHz}$ spur is from the CMBDSM sampling frequency. The two high-frequency dual-band spectra are the replica images of band-pass DSM, which also need to be suppressed by a dual-band RF band-pass filter.

\section{CONCLUSION}

An inter-band CA digital transmitter architecture with CMB-DSM (concurrent multi-band delta-sigma modulation) using out-of-band quantization noise cancellation technique is demonstrated for LTE transmission with 40-MHz aggregated bandwidth at $856 \mathrm{MHz}$ and $1450 \mathrm{MHz}$ channels. CMB-DSM allows multi-level digital Class-S PAs to achieve both good linearity and efficiency for inter-band CA. A digital loop-filter compensation technique is applied to CMB-DSM in order to avoid undesirable peaking in the noise transfer function when the frequency spacing between inter-band channels is narrow.

The out-of-band noise cancellation technique reduces the adjacent channel leakage and allows the use of a lessdemanding filter to suppress the out-of-band emission of CMB-DSM. The out-of-band quantization noise cancellation signal is created by a replica CMB-DSM, which compares the main CMB-DSM signal with the original multi-band baseband signals. The noise cancellation signal power is scaled down near the lowest power level of the main CMB-DSM signal. Hence, to achieve a high efficiency, the noise cancellation signal and the main CMB-DSM signal is summed by an asymmetric power combiner with unequal input power.

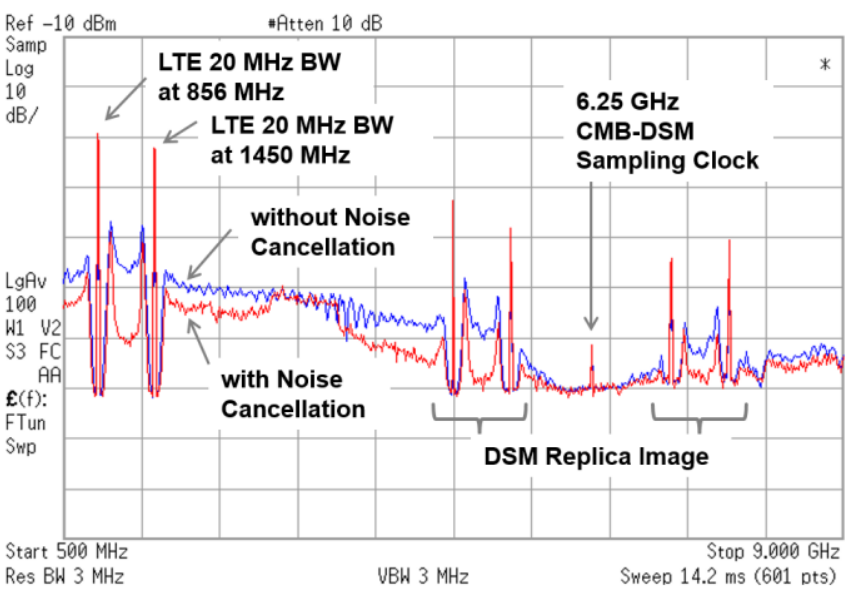

Fig. 9. Measured wideband output spectrum of the prototype CMBDSM digital transmitter with the LTE inter-band CA at $856 \mathrm{MHz}$ and $1450 \mathrm{MHz}$ channels.

\section{ACKNOWLEDGEMENT}

The authors would like to thank J. Shao at the University of North Texas, for his work on the asymmetric power combiner design during his internship at Mitsubishi Electric Research Laboratories. The authors would like to thank T. KoikeAkino, K. Parsons, and K. Yamanaka at Mitsubishi Electric for their advice and technical support.

\section{REFERENCES}

[1] X. Chen, W. Chen, and F. M. Ghannouchi, "Enhanced analysis and design method of concurrent dual-band power amplifiers with intermodulation impedance tuning," IEEE Trans. Microw. Theory Tech., vol. 61, no. 12, pp. 4544-4558, Dec. 2013.

[2] T. Kitayabu, Y. Amano, and H. Ishikawa, "Concurrent dual-band transmitter architecture for spectrum aggregation system," in Proc. IEEE Radio and Wireless Symposium, Jan. 2010, pp. 689-692.

[3] N. V. Silva, A. S. R. Oliveira, N. B. Carvalho, "Design and optimization of flexible and coding efficient all-digital RF transmitters," IEEE Trans. Microw. Theory Tech., vol. 61, no. 1, pp. 625-632, Jan. 2013.

[4] T. Maehata, K. Totani, S. Kameda, and N. Suematsu, "Concurrent dualband 1-bit digital transmitter using band-pass delta-sigma modulator," in Proc. IEEE European Microw. Conf., Oct. 2013, pp. 1523-1526.

[5] T.-P. Hung et al., "Design of H-bridge Class-D power amplifiers for digital pulse modulation transmitters," IEEE Trans. Microw. Theory Tech., vol. 55, no. 11, pp. 2845-2855, Dec. 2007.

[6] Q. Zhu et al., "A 5-level discrete-time power encoder with measured coding efficiency of $70 \%$ for $20-\mathrm{MHz}$ LTE digital transmitter," in Proc. IEEE MTT-S Int. Microw. Symp., June 2014.

[7] S. Chung, et al., "Concurrent multiband digital outphasing transmitter architecture using multidimensional power coding," IEEE Trans. Microw. Theory Techn., vol. 63, no. 2, pp. 598-613, Feb. 2015.

[8] H. H. Boo et al., "Adaptive predistortion using a delta-sigma modulator for automatic inversion of power amplifier nonlinearity," IEEE Trans. Circ. Syst. II: Express Papers, vol. 56, no. 12, pp. 901-905, Dec. 2009.

[9] R. Hezar et al., "A $23 \mathrm{dBm}$ fully digital transmitter using $\Delta \Sigma$ and pulsewidth modulation for LTE and WLAN application in 45nm CMOS," in Proc. IEEE Radio Freq. Integ. Circ. Symp., June 2014, pp. 217-220.

[10] L. I. Parad and R. L. Moynihan, "Split-tee power divider," IEEE Trans. Microw. Theory Techn., vol. 13, no. 1, pp. 91-95, Jan. 1965. 